\title{
Damoctocog Alfa Pegol: A Review in Haemophilia A
}

\author{
Julia Paik ${ }^{1} \cdot$ Emma D. Deeks ${ }^{1}$ \\ Published online: 19 June 2019 \\ (c) Springer Nature 2019, corrected publication 2019
}

\begin{abstract}
Damoctocog alfa pegol $\left(\mathrm{Jivi}^{\circledR}\right)$ is approved in the USA, EU, Japan and Canada for the treatment and prophylaxis of previously treated patients aged $\geq 12$ years with haemophilia A. Formulated with a $60 \mathrm{kDa}$ polyethylene glycol (PEG) moiety, damoctocog alfa pegol is an intravenously (IV) administered recombinant factor VIII (rFVIII) product with a longer terminal half-life than non-PEGylated FVIII and rFVIII products. In the multinational phase II/III PROTECT VIII trial, prophylaxis with damoctocog alfa pegol reduced the likelihood of bleeding in previously treated patients aged $\geq 12$ years with severe haemophilia $\mathrm{A}$, with dosing schedules ranging from twice weekly to once every 7 days. Interim data from the ongoing extension phase indicated that the reduced annualized bleeding rates (ABRs) were maintained for up to 5.2 years of prophylaxis with damoctocog alfa pegol. Damoctocog alfa pegol was also effective in treating bleeding episodes and in providing haemostatic control during surgery. Damoctocog alfa pegol was generally well tolerated in adult and adolescent patients with severe haemophilia A, with most adverse events considered to be unrelated to treatment. There were no new or confirmed cases of FVIII inhibitor development and anti-PEG antibodies, observed in some patients, were of low titre and transient. Damoctocog alfa pegol extends the available treatment options in previously treated adults and adolescents with haemophilia A, offering the possibility of up to once-weekly administration for suitable patients.
\end{abstract}

\section{Damoctocog alfa pegol: clinical considerations in haemophilia A}

IV PEGylated rFVIII designed to prolong FVIII activity; has a longer terminal half-life and greater exposure than non-PEGylated FVIII and rFVIII products

Prophylaxis reduced spontaneous and joint ABRs in previously treated adults and adolescents

Effective in treating bleeding episodes and for perioperative management

Generally well tolerated with no confirmed cases of antiFVIII inhibitor development

The manuscript was reviewed by: $\boldsymbol{J}$. Chapin, Clinical Development, CRISPR Therapeutics, Cambridge, MA, USA; $\boldsymbol{M}$. E. Mancuso, Fondazione IRCCS Ca' Granda, Ospedale Maggiore Policlinico, Angelo Bianchi Bonomi Hemophilia and Thrombosis Center, Milan, Italy; $\boldsymbol{P}$. Chowdary, Katharine Dormandy Haemophilia and Thrombosis Centre, Royal Free Hospital, London, UK; V. Horvais, Clinical Research Department, Nantes University Hospital, Nantes, France.

Julia Paik

demail@springer.com

1 Springer Nature, Private Bag 65901, Mairangi Bay, Auckland 0754, New Zealand

\section{Introduction}

Constituting $80-85 \%$ of the total haemophilia population, haemophilia $\mathrm{A}$ is an $\mathrm{X}$-linked congenital bleeding disorder involving coagulation factor VIII (FVIII) deficiency [1]. While individuals with mild (FVIII levels $5-40 \mathrm{IU} / \mathrm{dL}$ or 5 to $<40 \%$ of normal) or moderate (1-5 IU/dL, $1-5 \%$ of normal) haemophilia mostly experience bleeds with trauma or surgery, those with severe haemophilia $(<1 \mathrm{IU} / \mathrm{dL},<1 \%$ of normal) are more likely to experience spontaneous bleeding without any identifiable haemostatic challenges, most commonly in the joints (approximate frequency 70-80\%). Recurrent bleeding into the joints may result in irreversible haemophilic arthropathy, leading to chronic debilitating pain and subsequent disability [1].

With bleed prevention therefore being an important objective in haemophilia care, prophylactic intravenous (IV) replacement therapy with recombinant or plasma-derived FVIII products is the current mainstay approach for managing haemophilia A [2]. Prophylaxis can be optimized by tailoring the regimen to the individual, taking into account factors such as bleeding phenotype and pharmacokinetic profile [3]. However, the short circulating plasma half-life $\left(t_{1 / 2}\right)$ of FVIII (12-14 h) [4] often necessitates frequent dosing. Given that this affects treatment adherence due to reasons relating to convenience, cost or psychological impact (e.g. fear of needles) [5], a too-frequent dosing schedule may 
become a significant barrier to haemophilia management [3]. Another concern with FVIII replacement therapy is the potential for the development of neutralizing antibodies against the exogenous FVIII (i.e. inhibitors), which occurs in $\approx 30 \%$ of previously untreated patients with severe haemophilia A when treated with conventional FVIII [6]. Associated with substantial morbidity, inhibitor development is the most serious complication in haemophilia therapy and may cause greater incidences of bleeding complications, increased disability, and reduced health-related quality of life (HR-QOL) [7].

FVIII products are full-length or B-domain deleted (BDD) [8], which improves FVIII secretion from the cell during the recombinant process [9]. More recently developed recombinant FVIII (rFVIII) products (such as conjugating the FVIII to albumin or the human immunoglobulin Fc) have been designed to extend the $t_{1 / 2}$ to allow less frequent dosing [8]. Another approach is PEGylation, the attachment of a polyethylene-glycol (PEG) moiety to the FVIII molecule [10], which protects FVIII from removal from the plasma (Sect. 2). Although non-specific PEGylation extends the $t_{1 / 2}$ of FVIII, it may be at the cost of reduced activity, whereas strategic, site-specific PEGylation extends drug availability without compromising drug activity [11]. Moreover, the controlled PEG:FVIII molar ratio with site-specific PEGylation allows control over the amount of administered PEG, reducing the risk of possible PEG-related adverse effects from substantial amounts of high molecular-weight PEG (e.g. cellular vacuolation, although this has not been associated with any adverse effects in clinical studies) [12].

Damoctocog alfa pegol $\left(\mathrm{Jivi}^{\circledR}\right)$ is the first site-specifically PEGylated rFVIII product [13] approved in the USA [13], the EU [14], Japan [15] and Canada [16] for the treatment and prophylaxis of previously treated patients aged $\geq 12$ years with haemophilia A. This review discusses pharmacological, therapeutic efficacy and tolerability data relevant to the use of damoctocog alfa pegol in this setting.

\section{Pharmacodynamic Properties of Damoctocog Alfa Pegol}

Damoctocog alfa pegol is a BDD-rFVIII with a single, dualbranched $60 \mathrm{kDa}$ PEG moiety linked to a cysteine amino acid (via a maleimide linker) in the rFVIII A3 domain [17]. This site-specific PEGylation process extends the plasma $\mathrm{t}_{1 / 2}$ of the drug (Sect. 3) by reducing its binding affinity to FVIII clearance receptors [17], such as low-density lipoprotein receptor-related protein-1 (LRP1), which binds in the A3 (as well as the A2 [18] and C2 [19]) domain of FVIII [20]. Although the A3 domain may have a supporting role in the binding of FVIII with von Willebrand factor (VWF) [21], which stabilizes FVIII in the plasma and protects it from proteolysis [22], in vitro data demonstrated no significant differences in the maximum VWF binding capacity of damoctocog alfa pegol versus non-PEGylated BDD-FVIII or full-length rFVIII [11]. The rate of VWF association was reduced $\geq 3$.3-fold in damoctocog alfa pegol compared with non-PEGylated BDD-FVIII or full-length rFVIII; however, the VWF dissociation rate was not markedly altered [11].

The PEG moiety of damoctocog alfa pegol does not impact its function $[11,17]$. For instance, the drug can normalize activated partial thromboplastin time (aPTT; an in vitro indicator of FVIII activity) in haemophilia A patients (in whom aPTT is prolonged) to an extent similar to that seen with plasma-derived FVIII [17]. Although this is yet to be studied in haemophilia A patients with FVIII inhibitors, preclinical studies have suggested that the PEG moiety in damoctocog alfa pegol attenuates FVIII inhibitor binding, therefore facilitating retention of the drug's haemostatic function [11,23]. In assessed adults and adolescents from the phase II/III PROTECT VIII $(n=120)$ study [26] (see Sect. 4 for study details) and 52 children from another phase III study (PROTECT VIII Kids; $n=52$ ), the amount of delivered PEG with damoctocog alfa pegol prophylaxis was very low, with almost all PEG measurements below the lower limit of quantitation $(0.1 \mathrm{mg} / \mathrm{L})$ [24].

\section{Pharmacokinetic Properties of Damoctocog Alfa Pegol}

The pharmacokinetic parameters of IV damoctocog alfa pegol and sucrose-formulated rFVIII (rFVIII-FS) were assessed in a phase I crossover study in previously treated adults and adolescents with severe haemophilia A and no history of inhibitors (Table 1) [25]. The single-dose pharmacokinetic profile of damoctocog alfa pegol (25 or $60 \mathrm{IU} /$ $\mathrm{kg}$ ) showed that clearance was reduced 1.4-fold compared with that of sucrose-formulated rFVIII (rFVIII-FS) [25 and $50 \mathrm{IU} / \mathrm{kg}]$, in terms of the area under the FVIII activity-time curve (AUC), clearance and $t_{1 / 2}$ (Table 1) [25]. Moreover, the $t_{1 / 2}$ of damoctocog alfa pegol, when averaged across single and multiple doses of 25 and $60 \mathrm{IU} / \mathrm{kg}$, was significantly $(p<0.0001)$ longer than that of rFVIII-FS $(18.7$ vs. $\approx 13.0 \mathrm{~h})$ [25].

Damoctocog alfa pegol demonstrated dose-linear exposure in previously treated patients aged $\geq 12$ years with severe haemophilia A in both the phase I study [25] and the PROTECT VIII study [26], based on the mean peak plasma concentration $\left(\mathrm{C}_{\max }\right)$ and AUC after single doses of 25 and $60 \mathrm{IU} / \mathrm{kg}$ [17]. Other pharmacokinetic parameters, including recovery [i.e. ratio between $\mathrm{C}_{\max }$ (IU/dL) and administered dose (IU/kg); 2.13 and $2.53 \mathrm{IU} / \mathrm{dL}$ per IU $/ \mathrm{kg}$ after single doses of damoctocog alfa pegol 25 and $60 \mathrm{IU} / \mathrm{kg}$ ] were comparable between the two doses [17]. The pharmacokinetic 
parameters of damoctocog alfa pegol 25 or $60 \mathrm{IU} / \mathrm{kg}$ were shown to be mostly consistent after multiple doses (twiceweekly $25 \mathrm{IU} / \mathrm{kg}$ doses for 8 weeks [25]; once-weekly $60 \mathrm{IU} /$ $\mathrm{kg}$ doses for 9 weeks [25] and 36 weeks [27]) relative to those after a single dose. No clinically relevant differences in pharmacokinetic parameters were observed between adult and adolescent patients [27], with a population pharmacokinetics model $(n=145)$ supporting these findings [14].

Results from a population pharmacokinetics model based on data from participants in phase I, II and III studies ( $n=198$; aged 2-62 years) demonstrated the pharmacokinetics of damoctocog alfa pegol to be monophasic and adequately described by a one-compartment model [28]. The pharmacokinetics of damoctocog alfa pegol were similar between Japanese patients $(n=6)$ and non-Japanese patients $(n=18)$ [15].

Pharmacokinetic data in adult patients showed that VWF antigen levels had a significantly positive correlation with $\mathrm{t}_{1 / 2}$ and significantly negative correlation with clearance (both $p<0.0001$ ), indicating that damoctocog alfa pegol interacts with VWF to a similar degree to that of unmodified FVIII [27].

\section{Therapeutic Efficacy of Damoctocog Alfa Pegol}

The efficacy of on-demand treatment and routine prophylaxis with IV damoctocog alfa pegol in previously treated patients with haemophilia A was evaluated in the 36-week, partially randomized, open-label, multinational, phase II/ III PROTECT VIII trial (intent-to-treat population $n=132$ ) [26]. Eligible patients were males aged $12-65$ years with severe haemophilia A (FVIII < 1\%) who had previously been treated with any FVIII product(s) for $\geq 150$ exposure days. Patients with current evidence or a history of an inhibitor to FVIII ( $\geq 0.6 \mathrm{BU} / \mathrm{mL}$ ), a diagnosis of any bleeding disorder aside from haemophilia $\mathrm{A}$, a platelet count of $<100,000 / \mu \mathrm{L}$, creatinine levels $>2 \times$ the upper limit of normal (ULN), or aspartate aminotransferase (AST) or alanine aminotransferase (ALT) levels $>5 \times$ ULN were excluded from the study [26].

Patients who were receiving on-demand treatment prior to the study were able to choose between on-demand treatment and prophylaxis, while all patients receiving prophylaxis at the time of study enrolment were allocated into the prophylactic arm (Fig. 1) [26]. The prophylactic arm had a 10 -week run-in period in which patients were treated with twice-weekly damoctocog alfa pegol $25 \mathrm{IU} / \mathrm{kg}$. Afterwards, patients were allocated into different dosing groups for the main efficacy period of the study (i.e. weeks 11-36) depending on whether they had high bleeding tendencies [defined by $>1$ breakthrough bleed (joint or muscle bleeds and no identified trauma)] or good bleed control ( $\leq 1$ breakthrough bleed). Patients with good bleed control were eligible to be randomized to receive damoctocog alfa pegol every 5 days (starting dose $45 \mathrm{IU} / \mathrm{kg}$; increased to $60 \mathrm{IU} / \mathrm{kg}$ if their bleed control was inadequate) or 7 days (fixed dose $60 \mathrm{IU} / \mathrm{kg}$ ); once the every-5-days and every-7-days treatment arms were full, 11 patients eligible for randomization were instead given twice-weekly damoctocog alfa pegol $30-40 \mathrm{IU} / \mathrm{kg}$ (henceforth referred to as the twice-weekly eligible but not randomized group). Those with high bleeding tendencies were not eligible for randomization and were administered twice-weekly damoctocog alfa pegol 30-40 IU/kg (henceforth referred to as the twice-weekly non-eligible for randomization group) [Fig. 1] [26].

Treatment adjustments were recommended in the prophylaxis arms if patients experienced at least two joint and/or muscle breakthrough bleeds within any 10-week period [26]. If patients in the every-5-days treatment group had inadequate bleed control after increasing their dose to $60 \mathrm{IU} / \mathrm{kg}$, they were permitted a one-time change to twice-weekly dosing. Patients experiencing inadequate bleed control during every-7-days treatment were permitted to change to every5-days or twice-weekly treatment [26].

The primary efficacy endpoint was the annualized number of total bleeds [assessed as annualized bleeding rate

Table 1 Mean plasma pharmacokinetic parameters following a single intravenous dose of damoctocog alfa pegol or rFVIII-FS in previously treated patients aged $\geq 12$ years with severe haemophilia $A$ and no history of inhibitors $(n=14)$ [25]

\begin{tabular}{llllrrrr} 
Treatment & Dose $(\mathrm{IU} / \mathrm{kg})$ & $\mathrm{t}_{1 / 2}(\mathrm{~h})$ & $\mathrm{AUC}(\mathrm{IU} \cdot \mathrm{h} / \mathrm{dL})$ & $\mathrm{C}_{\text {max }}(\mathrm{IU} / \mathrm{dL})$ & $\mathrm{V}_{\mathrm{ss}}(\mathrm{dL} / \mathrm{kg})$ & $\mathrm{CL}(\mathrm{dL} / \mathrm{h} / \mathrm{kg})$ \\
\hline $\begin{array}{l}\text { Damoctocog alfa } \\
\text { pegol }\end{array}$ & 25 & 18.2 & 1577 & 63.7 & 0.43 & 0.016 \\
\hline rFVIII-FS & 60 & 18.5 & 4329 & 172.0 & 69.6 & 0.38 & 0.014 \\
& 25 & 12.9 & 1109 & 228.6 & 0.36 & 0.023 \\
\hline
\end{tabular}

FVIII activity measured using a chromogenic assay; all values are geometric means

$A U C$ area under the FVIII activity-time curve, $C_{\text {max }}$ peak plasma concentration, $C L$ clearance, $r F V I I I-F S$ sucrose-formulated recombinant factor VIII, $t_{1 / 2}$ plasma half-life, $V_{s s}$ volume of distribution at steady state 


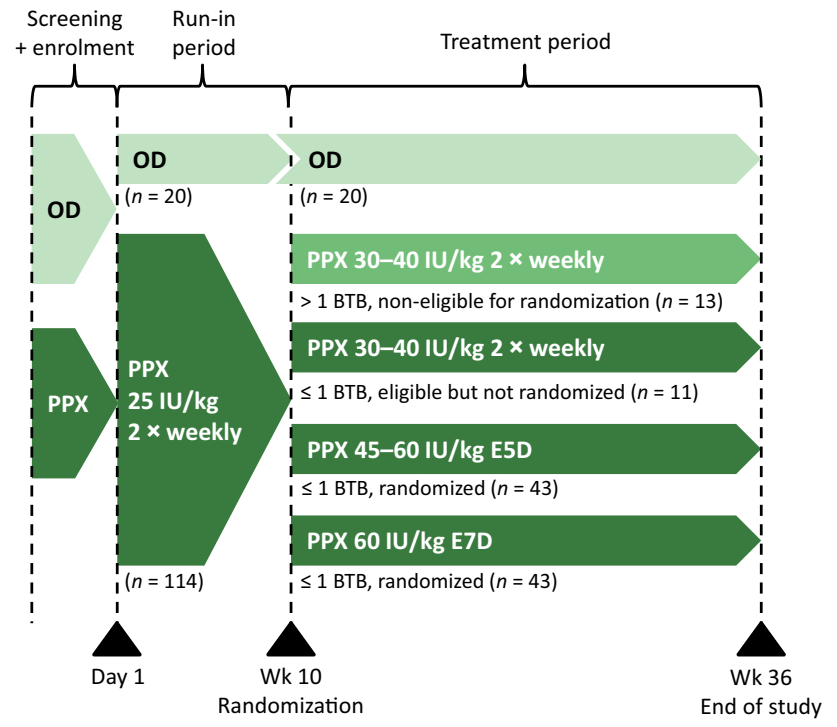

Fig. 1 Clinical study design of PROTECT VIII. Patients in the prophylaxis arm were allocated to different dosing groups according to the number of breakthrough bleeds experienced during the run-in period. $B T B$ breakthrough bleed, E5D every 5 days, E7D every 7 days, $O D$ on-demand treatment, $P P X$ prophylaxis. Adapted from Reding et al. [26] with permission

$(\mathrm{ABR})]$; secondary endpoints included patient assessment of response (rated "excellent", "good", "moderate" or "poor"), and the number of infusions, in the treatment of bleeding episodes [26].

An optional extension study assessing the long-term efficacy and safety of damoctocog alfa pegol in patients from the PROTECT VIII trial $(n=121)$ is currently ongoing (Sect. 4.1.1) [29]. Prophylaxis patients could either continue their regimen from the main study or change to another prophylaxis regimen, and on-demand patients could continue on-demand treatment or switch to a prophylaxis regimen [29].

The efficacy and safety of damoctocog alfa pegol as perioperative treatment were assessed in PROTECT VIII participants requiring surgery [30]. The study was also open to patients not participating in PROTECT VIII but who required surgery and met the PROTECT VIII inclusion criteria (PROTECT VIII Part B; discussed in Sect. 4.3) [31].

\subsection{Routine Prophylaxis}

Prophylaxis with damoctocog alfa pegol using any of the three dosing regimens was effective in reducing the likelihood of bleeding in previously treated patients with severe haemophilia A in PROTECT VIII. During the main efficacy period, the median total ABR was noticeably lower $(\geq 5.9$ fold) in the prophylaxis arms than in the on-demand treatment arm, with the median ABRs for spontaneous and joint bleeds supporting these findings (Table 2) [26]. Moreover, when the statistical estimate of effect was assessed post hoc, mean ABRs were significantly $(p<0.0001)$ lower with each of the prophylactic regimens versus on-demand treatment (75-92\% reduction) [31]. In the same analysis, the ABRs from twice-weekly treatment did not significantly differ to those of the extended interval treatments [31].

Throughout weeks $0-36$, a median of 2.0 bleeds (range $0-17)$ was reported in the 110 patients in the entire prophylaxis arm versus 15.5 bleeds (range 5-59) in the 20 patients in the on-demand treatment arm [13]. Among all prophylaxis recipients, 38\% did not experience any bleeds during the main efficacy phase of the study, with the highest proportion of patients with zero bleeds (46\%) seen in the twice-weekly eligible but not randomized group [26]. All patients in the on-demand treatment arm experienced at least one bleeding episode [17].

While all patients in the every-5-days treatment group maintained their dose frequency throughout the main efficacy period, 11 patients left the every-7-days treatment group for a higher frequency dosing group after a mean of 84 days (with eight patients moving to every-5-days treatment and three patients moving to twice-weekly treatment) [26]. The median ABRs in these 11 patients were 16.9 and 4.7 before and after leaving the group; three (27\%) experienced no bleeding episodes after switching to more frequent dosing. The median ABR in the 32 patients who continued every-7-days treatment was $0.96 ; 16(50 \%)$ had no bleeding episodes. These findings, together with the fact that the median total ABR markedly improved in the twice-weekly non-eligible for randomization group in the main efficacy phase (average dose $38.9 \mathrm{IU} / \mathrm{kg}$ ) versus the run-in phase (fixed dose $25 \mathrm{IU} / \mathrm{kg}$ ) [Table 2], further support the benefit of appropriately individualized damoctocog alfa pegol prophylaxis dosing in this setting [26].

Post hoc subgroup analyses of the PROTECT VIII study supported the efficacy of adjusting damoctocog alfa pegol prophylaxis dosing depending on the bleeding phenotype of the patient, with the number of total bleeds in the last 12 months and the number of target joints at baseline identified as possible indicators of suitability for every-5-days or every-7-days dosing (abstracts) [32-34]. Among every-5days recipients, those with zero bleeds during the trial had fewer bleeds in the 12 months prior to the study than those with an $\mathrm{ABR} \geq 1$ (median total bleeds 2 vs. 10), and fewer zero-bleed patients had target joints at baseline than ABR $\geq 1$ patients (58 vs. $71 \%$ of patients) [abstract data] [34]. Similarly, among patients receiving every-7-days treatment, those with zero bleeds during the trial had fewer total bleeds in the 12 months prior to the study (median 2 vs. 3.5) and fewer target joints at baseline (median 1 vs. 2) than those who switched to more frequent dosing groups [33]. 
Table 2 Efficacy of damoctocog alfa pegol in the PROTECT VIII phase II/III study in previously treated male patients aged 12-65 years with severe haemophilia A

\begin{tabular}{|c|c|c|c|c|c|c|c|}
\hline \multirow[t]{2}{*}{ Treatment } & \multirow[t]{2}{*}{ Dosing interval } & \multirow{2}{*}{$\begin{array}{l}\text { No. of } \\
\text { mITT pts }\end{array}$} & \multirow{2}{*}{$\begin{array}{l}\text { Run-in } \mathrm{ABR}^{\mathrm{a}} \\
\text { (median) }\end{array}$} & \multicolumn{3}{|c|}{ Median ABR } & \multirow{2}{*}{$\begin{array}{l}\text { Average dose } \mathrm{e}^{\mathrm{b}} \\
\text { (IU/kg per infusion }\end{array}$} \\
\hline & & & & Total $^{\mathrm{c}}$ & Joint & Spontaneous & \\
\hline \multicolumn{8}{|c|}{ Main study ${ }^{d}[26,29]$} \\
\hline On-demand & & 20 & & 23.4 & 16.3 & 14.3 & 35.5 \\
\hline \multirow[t]{5}{*}{ Prophylaxis } & Twice weekly, non-eligible for randomization & 13 & 17.4 & 4.1 & 4.0 & 3.9 & 38.9 \\
\hline & Twice weekly, eligible but not randomized & 11 & 0 & 1.9 & 1.9 & 0 & 31.5 \\
\hline & Every 5 days & 43 & 0 & 1.9 & 1.9 & 0 & 45.3 \\
\hline & Every 7 days ${ }^{\mathrm{e}}$ & 43 & 0 & 3.9 & 1.9 & 1.9 & 56.8 \\
\hline & Total prophylaxis & 110 & & 2.1 & 1.9 & 0 & \\
\hline \multicolumn{8}{|c|}{ Extension study ${ }^{\mathrm{f}}[29]$} \\
\hline On-demand & & 14 & & 33.7 & 20.5 & 20.7 & 32.8 \\
\hline \multirow[t]{5}{*}{ Prophylaxis } & Twice weekly & 23 & & 1.8 & 0.8 & 0.8 & 36.7 \\
\hline & Every 5 days & 34 & & 1.3 & 1.0 & 0.7 & 45.1 \\
\hline & Every 7 days & 23 & & 0.7 & 0.3 & 0 & 59.3 \\
\hline & Variable frequency ${ }^{\mathrm{g}}$ & 27 & & 3.2 & 2.4 & 1.9 & 52.1 \\
\hline & Total prophylaxis & 107 & & 1.6 & 0.9 & 0.8 & 46.6 \\
\hline
\end{tabular}

$A B R$ annualized bleeding rate, $m I T T$ modified intention-to-treat, $p t s$ patient

${ }^{a}$ Weeks 1-10; fixed twice-weekly dose of $25 \mathrm{IU} / \mathrm{kg}$ per infusion

${ }^{\mathrm{b}}$ Mean dose reported in main phase of the main study; median dose reported in extension phase

${ }^{c}$ Primary endpoint in main efficacy phase; weeks 0-36 in on-demand treatment arm, weeks 11-36 for prophylaxis arms

${ }^{\mathrm{d}}$ Some data for spontaneous and joint ABRs and average dose are reported from other sources [13, 17, 31]

${ }^{\mathrm{e}}$ Includes data from pts that remained in $(n=32)$ and pts that left $(n=11)$ the treatment arm

${ }^{\mathrm{f}}$ Data assessed over median duration of 166 weeks in extension study (data cut-off August 2017)

${ }^{\mathrm{g}}$ Includes pts who switched dosing regimens during the extension phase

Prophylactic treatment with damoctocog alfa pegol improved HR-QOL in previously treated patients (abstract data) [35]. At week 36, an improvement was seen in the assessed prophylaxis patients $(n=97)$ [median total change in Haemo-QoL-A + 2.5 from baseline], with the greatest improvements seen in patients with prior on-demand treatment who received every-7-days prophylaxis in the study $(n=5$; change in median total score +13.8$)$. No improvements were observed in patients in the on-demand treatment $\operatorname{arm}[35]$.

\subsubsection{Extension Phase}

Interim data (data cut-off August 2017) from 121 patients who entered the extension phase of the PROTECT VIII study indicated that bleed control was well maintained with prophylactic treatment with damoctocog alfa pegol for up to 5.2 years (abstract plus poster) [29]. At the time of analysis, most patients (90.9\%) accumulated at least 100 exposure days. Median ABRs remained generally consistent with those reported in the main phase in all treatment groups, with lower median total, spontaneous and joint ABRs maintained in all prophylaxis dosing groups relative to the on-demand group (median total ABR $\geq 10.5$-fold lower) [Table 2]. The median doses per infusion reported in the extension study were also comparable to the mean doses in the main study (Table 2) [29].

According to a post hoc analysis of earlier interim data (data cut-off February 2017), prophylaxis patients experienced a numerical decrease in median ABR from before the main phase of PROTECT VIII to the extension study (e.g. median ABR 3.5 before the main study, 2.1 during the main study, and 1.6 during the extension study in all 82 evaluable prophylaxis patients), despite some of the prophylaxis regimens having been taken more frequently (i.e. 3 or 4 times per week) [abstract plus poster] [36].

More recent interim data (data cut-off January 2018) have indicated that the $\mathrm{ABR}$ and joint $\mathrm{ABR}$ in the last 12 months of the extension phase (overall median $A B R$ and joint $A B R$ 1.0 and $<1.0$ across prophylaxis groups) [37] have remained consistent with those reported earlier (data cut-off August 2017; Table 2). In addition, annual damoctocog alfa pegol consumption in prophylaxis patients was similar in the main and extension studies and across dosage groups (median FVIII consumption 3339-3946 IU/kg/year in the main study, 3120-3917 IU/kg/year in the extension study), indicating 
that low ABRs were maintained without requiring increased FVIII consumption [37].

\subsection{Treatment of Bleeding Episodes}

Bleeding episodes (388 and 317 total treated bleeds in the on-demand and prophylaxis groups) were generally well controlled with damoctocog alfa pegol [17]. The majority of bleed treatments in the on-demand and prophylaxis groups (66 and 83\%) were considered either "excellent" or "good"; across both groups, $3.3 \%$ of bleed treatments had "poor" responses. In most bleed cases, haemostasis was achieved with one infusion in the on-demand $(80 \%)$ and prophylaxis (83\%) groups; two infusions were required in 12 and $7 \%$ of bleed cases and at least three infusions were needed in 9 and $10 \%$ of bleed cases [17]. The mean dose per infusion in bleed treatment was 33.7 (range 14-62) IU/kg and the mean length of time between the first and second infusion was 2.1 days [26]. An interim analysis of the extension study indicated that most of the 514 and 428 total bleeds in the 14 and 107 on-demand treatment (514 bleeds in 14 patients) and prophylaxis ( 428 bleeds in 107 patients) groups were successfully treated with one or two infusions [17].

\subsection{Perioperative Management in Major Surgery}

Interim data suggest that in patients with severe haemophilia A, damoctocog alfa pegol is effective in providing haemostatic control during major surgery. In total, 17 patients (aged 13-61 years) completed 20 major surgeries [17], with a median surgical duration (range) of 102 (17-217) $\mathrm{min}$ (abstract data) [30]. Of these, 15 were orthopaedic surgeries [30]. On the day of surgery, the median damoctocog alfa pegol dose used was $72.4 \mathrm{IU} / \mathrm{kg}$ (median presurgical dose $52.1 \mathrm{IU} / \mathrm{kg}$ ), with a median of two infusions (median time between presurgical and second infusions $12.3 \mathrm{~h}$ ); $40 \%$ of surgeries required only one infusion (preoperative) on the day of surgery [30]. In all 20 major surgeries, treatment with damoctocog alfa pegol provided "good" (65\% of surgeries) or "excellent" (35\%) haemostatic control [30].

\section{Tolerability of Damoctocog Alfa Pegol}

Damoctocog alfa pegol was generally well tolerated in previously treated patients aged $\geq 12$ years with severe haemophilia A in phase I (Sect. 3) [25] and phase II/III (PROTECT VIII; Sect. 4) [26] clinical trials. In a pooled analysis of the two studies ( $n=148$; median 131 exposure days), which excluded those who received damoctocog alfa pegol for perioperative management, the most common adverse events (AEs) were headache (14\%), cough (7\%), nausea (5\%) and fever (5\%) [17].

In PROTECT VIII, patients accumulated a mean of 55 exposure days, with $69.4 \%$ of patients accumulating $\geq 50$ exposure days [26]. Treatment-related AEs (TRAEs) occurred in 12 patients (9\%), and included dry mouth, hypersensitivity, pruritus (including vessel site pruritus), "fuzzy thinking", arthralgia, palpitations, overdose, increased transaminases, abdominal pain and dyspnea [13]. Four serious AEs (SAEs) occurred in four patients in the on-demand group (ethanol intoxication, pneumonia, ankle sprain and acute pancreatitis) and 11 SAEs occurred in 10 patients in the prophylaxis group (including drug hypersensitivity, tendon rupture, bile duct stone, haemarthrosis, chest pain, gastroenteritis, injury, arthropathy and overdose), all of which were considered probably (in the case of hypersensitivity) or possibly related to study drug [13]. Two patients withdrew from the study due to brief and self-limited systemic hypersensitivity reactions (after the first or fourth exposure) [26].

When used in perioperative management in PROTECT VIII, four treatment-related SAEs [non-cardiac chest pain (1 event), haemorrhage/haematoma (1 event) and antiFVIII antibodies (2 events; see also Sect. 5.1)] were reported in 3 of the 16 evaluated patients (14 surgical and 2 nonsurgical patients) [13].

The tolerability profile of damoctocog alfa pegol was largely consistent for up to 5.2 years of treatment, according to findings from the PROTECT VIII extension phase, and no new safety issues were identified [13, 29, 38]. Among the patients in the PROTECT VIII extension study ( $n=121$; median 223 exposure days), $90.9 \%$ of patients had $\geq 100$ exposure days at the time of analysis (data cut-off August 2017) [29].

\subsection{Immunogenicity}

When immunogenicity was assessed in 159 previously treated adults and adolescents in the phase I and PROTECT VIII studies (median 131 exposure days), there were no new or confirmed cases of FVIII inhibitor development with damoctocog alfa pegol [14], including in the PROTECT VIII extension phase [38]. Two patients receiving damoctocog alfa pegol for perioperative management in the PROTECT VIII study tested positive for low titre anti-FVIII antibodies [13]. In one patient, the positive anti-FVIII titre observed after surgery was unconfirmed and of low titre (1.7 BU/mL) [17].

Five patients who tested negative for anti-PEG nonneutralizing antibodies (NNAs) at baseline tested positive during the main phase of the PROTECT VIII study [26]. However, no AEs or any adverse effects relating to bleeding or dose were associated with these NNAs, and all patients 
tested negative at the final visit. An anti-PEG immunoglobulin $\mathrm{M}$ antibody was detected 4 days after the hypersensitivity reaction in one of the two patients who withdrew from the PROTECT VIII study (Sect. 5); however, it was low titre and was undetectable after a month [26]. The anti-PEG antibodies detected in eight patients during the extension phase of the PROTECT VIII study were transient, low-titre, and not associated with any clinical events [38].

\section{Dosage and Administration of Damoctocog Alfa Pegol}

Damoctocog alfa pegol is approved in the USA [17], the EU [14], Japan [15] and Canada [16] for the prophylaxis, perioperative management and on-demand treatment (and control [17]) of bleeding in previously treated patients aged $\geq 12$ years with haemophilia A. It is administered intravenously, with the dose injected over $1-15 \min [16,17]$ or $2-5 \mathrm{~min}$ [14] at a rate no greater than $2.5 \mathrm{~mL} / \mathrm{min}$; the rate of administration depends on the response $[16,17]$ and comfort [14] of the patient.

The goal of therapy in on-demand and perioperative bleeding management is to maintain a plasma FVIII at or above a certain level determined by the extent of bleeding or type of surgery, and the dosage and duration of treatment depend on the level of FVIII deficiency, the location and severity of bleeding, and the clinical condition of the patient $[14,15,17]$.

The recommended regimens for prophylaxis differ by region, with the regimen selected and/or adjusted on the basis of individual patient characteristics (in the EU [14], Japan [15] and Canada [16]) and response to treatment (the USA [17], EU [14] and Canada [16]). In the USA and Canada, the recommended initial dosage is $30-40 \mathrm{IU} / \mathrm{kg}$ twice weekly which, based on bleeding episodes, may be adjusted to $45-60 \mathrm{IU} / \mathrm{kg}$ every 5 days or further adjusted on an individual basis $[16,17]$. The recommended regimen in the EU is $45-60 \mathrm{IU} / \mathrm{kg}$ once every 5 days, which may be adjusted to $60 \mathrm{IU} / \mathrm{kg}$ every 7 days or 30-40 IU/kg twice weekly depending on the clinical characteristics of the patient [14]. The maximum dose per infusion for should not exceed $\approx 6000 \mathrm{IU}$ [17] (for prophylaxis in overweight patients in the EU [14]). In Japan, the usual dose for routine prophylaxis is 30-40 IU/ $\mathrm{kg}$ twice weekly, which may be adjusted to $45-60 \mathrm{IU} / \mathrm{kg}$ once every 5 days or $60 \mathrm{IU} / \mathrm{kg}$ once weekly depending on the patient's conditions [15].

Local prescribing information should be consulted for detailed information including contraindications, precautions, drug interactions and use in special patient populations and FVIII monitoring recommendations.

\section{Current Status of Damoctocog Alfa Pegol in Haemophilia A}

The prevention and treatment of bleeding episodes, as well as maintaining haemostasis in surgical settings, is paramount in the management of haemophilia A [1, 39]. The primary strategy in the management of haemophilia A is regular IV replacement therapy (i.e. prophylaxis) with FVIII, which facilitates the maintenance of normal musculoskeletal function by minimizing joint bleeds and potential arthropathy $[1,39]$. While much less common, bleeds outside of the musculoskeletal system, such as intracranial haemorrhages, can be managed by secondary prophylaxis [1]. Many FVIII products have been developed and made available over time, with the abundance of choices complicating the process of selecting the appropriate product for a patient [8].

Frequent administration is often inevitable with conventional FVIII products due to their short $t_{1 / 2}$ and it is a known barrier to treatment adherence (Sect. 1). Products with extended $t_{1 / 2}$ require less frequent administration and may thus be a prudent choice, particularly for patients with poor compliance [8]. However, the potential benefits of FVIII products with longer $t_{1 / 2}$ are not limited to the patient; caregiver burden has also been shown to be lessened with factor products with extended $t_{1 / 2}$ compared with conventional products [40]. Currently, the only other approved PEGylated FVIII products aside from damoctocog alfa pegol are rurioctocog alfa pegol [41, 42] and turoctocog alfa pegol [43]; however, damoctocog alfa pegol is the only FVIII product that offers the option of once-weekly treatment. The sitespecific PEGylation in damoctocog alfa pegol facilitates a longer $t_{1 / 2}$ of the drug without compromising its activity, allowing less frequent administration (Sects. 3 and 6).

In the PROTECT VIII study, damoctocog alfa pegol was effective in the prophylaxis and treatment of bleeding episodes, including for perioperative management, in previously treated adults and adolescents with severe haemophilia A (Sect. 4). Prophylaxis with damoctocog alfa pegol reduced the likelihood of bleeding at dosing intervals ranging from twice weekly to every 7 days, with interim extension phase data indicating that these benefits may be maintained for up to 5.2 years of treatment. Proportionally, bleeding episodes were more frequent in the on-demand treatment arm than in the prophylaxis arm (Sect. 4.1). Most bleeding episodes were well controlled (the majority with one infusion) [Sect. 4.2] and favourable haemostatic control was attained with damoctocog alfa pegol in all major surgeries (Sect. 4.3).

Damoctocog alfa pegol was generally well tolerated in adult and adolescent patients for up to 5.2 years of treatment (Sect. 5). TRAEs and SAEs were uncommon in the PROTECT VIII study, and the latest data from the extension 
phase showed that all but one of the reported TRAEs were mild or moderate in severity and all of the reported SAEs were resolved or recovering. Although two patients withdrew from the PROTECT VIII study due to hypersensitivity reactions, there were no confirmed cases of inhibitor development against damoctocog alfa pegol; anti-PEG antibody titres were low (where reported) and transient (Sect. 5.1), which is consistent with preclinical toxicology studies demonstrating the lack of toxicity of proteins with larger PEG moieties [10].

Long-term data, including the completion of the PROTECT VIII extension phase, will be beneficial in determining the durability of the efficacy and safety of damoctocog alfa pegol. Although current studies have not reported PEGrelated AEs with damoctocog alfa pegol, data relevant to the long-term safety of the larger PEG moiety would be valuable, with events such as cellular vacuolation linked with drugs with larger PEG in nonclinical studies [12]. A recent analysis suggested that damoctocog alfa pegol had comparable efficacy to that of three other rFVIII products (efmoroctocog alfa, BAX 855 and rAHF-PFM), and the mean weekly drug consumption was significantly (vs. efmoroctocog alfa; $p<0.001$ ) or numerically lower (vs. BAX 855 and rAHFPFM) with damoctocog alfa pegol (abstract data) [44]. Moreover, AUC-related findings from a head-to-head study in patients with severe haemophilia A have suggested that damoctocog alfa may have an improved pharmacokinetic profile than $\mathrm{rFVIIIFc} \mathrm{[45].} \mathrm{Further} \mathrm{head-to-head} \mathrm{trials} \mathrm{are}$ needed to more definitively position damoctocog alfa pegol among other available treatments for haemophilia A. Costeffectiveness analyses are required to determine whether the extended $t_{1 / 2}$ of damoctocog alfa pegol, and therefore the expected lower dose frequency, supports the cost of the product.

To conclude, damoctocog alfa pegol is effective in the prophylaxis and treatment of bleeding episodes (as well as in perioperative management) and is generally well tolerated in previously treated patients aged $\geq 12$ years with haemophilia A. With its formulation facilitating a longer systemic presence, damoctocog alfa pegol addresses a well-known problem in FVIII replacement therapy without compromising therapeutic effect. While further evidence will help to more definitively establish the place of damoctocog alfa pegol in haemophilia A management, damoctocog alfa pegol extends the available treatment options in previously treated adults and adolescents with haemophilia A, offering the possibility of up to once-weekly dosing for suitable patients.

\section{Data Selection Damoctocog alfa: 125 records} identified

Duplicates removed

Excluded during initial screening (e.g. press releases; news reports; not relevant drug/indication; preclinical study; reviews; case reports; not randomized trial)

Excluded during writing (e.g. reviews; duplicate data; small patient number; nonrandomized/phase I/II trials)

Cited efficacy/tolerability articles

Cited articles not efficacy/tolerability

Search Strategy: EMBASE, MEDLINE and PubMed from 1946 to present. Clinical trial registries/databases and websites were also searched for relevant data. Key words were damoctocog alfa, Jivi, BAY 94-9027, haemophilia A. Records were limited to those in English language. Searches last updated 6 June 2019

Acknowledgements During the peer review process, the manufacturer of damoctocog alfa pegol was also offered an opportunity to review this article. Changes resulting from comments received were made on the basis of scientific and editorial merit.

\section{Compliance with Ethical Standards}

Funding The preparation of this review was not supported by any external funding.

Conflicts of interest Julia Paik and Emma Deeks are salaried employees of Adis International Ltd/Springer Nature, are responsible for the article content and declare no relevant conflicts of interest.

Open Access This article is distributed under the terms of the Creative Commons Attribution-NonCommercial 4.0 International License (http://creativecommons.org/licenses/by-nc/4.0/), which permits any noncommercial use, duplication, adaptation, distribution and reproduction in any medium or format, as long as you give appropriate credit to the original author(s) and the source, provide a link to the Creative Commons license and indicate if changes were made.

\section{References}

1. Srivastava A, Brewer AK, Mauser-Bunschoten EP, et al. Guidelines for the management of hemophilia. Haemophilia. 2013;19(1):e1-47.

2. Srivastava A. Haemophilia care: beyond the treatment guidelines. Haemophilia. 2014;20(Suppl 4):4-10.

3. Petrini P, Valentino LA, Gringeri A, et al. Individualizing prophylaxis in hemophilia: a review. Expert Rev Hematol. 2015;8(2):237-46.

4. Bjorkman S, Folkesson A, Jonsson S. Pharmacokinetics and dose requirements of factor VIII over the age range 3-74 years: a population analysis based on 50 patients with long-term 
prophylactic treatment for haemophilia A. Eur J Clin Pharmacol. 2009;65(10):989-98.

5. Saxena K. Barriers and perceived limitations to early treatment of hemophilia. J Blood Med. 2013;4:49-56.

6. Scharrer I, Bray GL, Neutzling O. Incidence of inhibitors in haemophilia A patients: a review of recent studies of recombinant and plasma-derived factor VIII concentrates. Haemophilia. 1999;5(3):145-54.

7. Witmer C, Young G. Factor VIII inhibitors in hemophilia A: rationale and latest evidence. Ther Adv Hematol. 2013;4(1):59-72.

8. Lieuw K. Many factor VIII products available in the treatment of hemophilia A: an embarrassment of riches? J Blood Med. 2017;8:67-73.

9. Miao HZ, Sirachainan N, Palmer L, et al. Bioengineering of coagulation factor VIII for improved secretion. Blood. 2004;103(9):3412-9.

10. Ivens IA, Baumann A, McDonald TA, et al. PEGylated therapeutic proteins for haemophilia treatment: a review for haemophilia caregivers. Haemophilia. 2013;19(1):11-20.

11. Mei B, Pan C, Jiang H, et al. Rational design of a fully active, long-acting PEGylated factor VIII for hemophilia A treatment. Blood. 2010;116(2):270-9.

12. Ivens IA, Achanzar W, Baumann A, et al. PEGylated biopharmaceuticals: current experience and considerations for nonclinical development. Toxicol Pathol. 2015;43(7):959-83.

13. US Food and Drug Administration. JIVI (antihemophilic factor (recombinant), PEGylated-aucl): clinical review. 2018. https:// www.fda.gov/. Accessed 6 June 2019.

14. European Medicines Agency. Damoctocog alfa pegol (Jivi): EU summary of product characteristics. 2018. http://www.ema.europ a.eu. Accessed 6 June 2019.

15. Pharmaceuticals and Medical Devices Agency. Jivi ${ }^{\circledR}$ for IV injection: Japanese prescribing information. 2018. http://www.pmda. go.jp/. Accessed 6 June 2019

16. Bayer HealthCare LLC. JIVI ${ }^{\circledR}$ [antihemophilic factor (recombinant), B-domain deleted, PEGylated]: Canadian product monograph. 2018. https://www.bayer.ca/. Accessed 6 June 2019.

17. Bayer HealthCare LLC. JIVI ${ }^{\circledR}$ [antihemophilic factor (recombinant), PEGylated-aucl]: US prescribing information. 2018. https ://www.fda.gov/. Accessed 6 June 2019.

18. Saenko EL, Yakhyaev AV, Mikhailenko I, et al. Role of the low density lipoprotein-related protein receptor in mediation of factor VIII catabolism. J Biol Chem. 1999;274(53):37685-92.

19. Lenting PJ, Neels JG, van den Berg BM, et al. The light chain of factor VIII comprises a binding site for low density lipoprotein receptor-related protein. J Biol Chem. 1999;274(34):23734-9.

20. Bovenschen N, Boertjes RC, van Stempvoort G, et al. Low density lipoprotein receptor-related protein and factor IXa share structural requirements for binding to the $\mathrm{A} 3$ domain of coagulation factor VIII. J Biol Chem. 2003;278(11):9370-7.

21. Chiu PL, Bou-Assaf GM, Chhabra ES, et al. Mapping the interaction between factor VIII and von Willebrand factor by electron microscopy and mass spectrometry. Blood. 2015;126(8):935-8.

22. Weiss HJ, Sussman II, Hoyer LW. Stabilization of factor VIII in plasma by the von Willebrand factor. Studies on posttransfusion and dissociated factor VIII and in patients with von Willebrand's disease. J Clin Invest. 1977;60(2):390-404.

23. Yada K, Nogami K, Patel C, et al. A factor VIII with sitespecific PEGylation attenuates the binding with inhibitors results in the retention of hemostatic effect [abstract]. Blood. 2015;126(23): 1076

24. Maas Enriquez M, Katterle Y, Baumann A, et al. BAY 94-9027 PROTECT VIII studies: observation of pharmacokinetic steady state for $60-\mathrm{kDa}$ PEG demonstrates the existence of elimination processes for PEG [abstract no. T-FPMED02-002 (600)]. Haemophilia. 2018;24(Suppl 5):16

25. Coyle TE, Reding MT, Lin JC, et al. Phase I study of BAY 94-9027, a PEGylated B-domain-deleted recombinant factor VIII with an extended half-life, in subjects with hemophilia A. J Thromb Haemost. 2014;12(4):488-96.

26. Reding MT, Ng HJ, Poulsen LH, et al. Safety and efficacy of BAY 94-9027, a prolonged-half-life factor VIII. J Thromb Haemost. 2017;15(3):411-9.

27. Shah A, Coyle T, Lalezari S, et al. BAY 94-9027, a PEGylated recombinant factor VIII, exhibits a prolonged half-life and higher area under the curve in patients with severe haemophilia A: comprehensive pharmacokinetic assessment from clinical studies. Haemophilia. 2018;24(5):733-40.

28. Ahsman M, Vis P, Shah A, et al. Predictable and reliable individualized pharmacokinetic profiling with BAY 94-9027: integrated population pharmacokinetics analysis [abstract no. T-P092 (372)]. Haemophilia. 2018;24(Suppl 5):89-90.

29. Thomson G, Holme PA, Wang M, et al. Effective protection for $>5$ years with BAY 94-9027 prophylaxis: interim results from the PROTECT VIII extension trial [abstract plus poster]. In: The 11th Annual Congress of the European Association for Haemophilia and Allied Disorders. 2018.

30. Santagostino E, Lalezari S, Ducore J, et al. BAY 94-9027 is efficacious in maintaining hemostasis during major surgery in adults and adolescents with severe hemophilia A: PROTECT VIII results [abstract no. P079]. Haemophilia. 2018;24(Suppl 1):69.

31. European Medicines Agency. Jivi (damoctocog alfa pegol): public assessment report. 2019. https://www.ema.europa.eu/. Accessed 6 June 2019

32. Oldenburg J, Michaels LA, Wang M, et al. PROTECT VIII: can patient characteristics predict eligibility for less frequent prophylaxis dosing regimens? [abstract no. PB 1818]. Res Pract Thromb Haemost. 2017;1(Suppl 1):807-8.

33. Holme PA, Wang M, Saxena K, et al. Predictors of patients with 0 bleeds during every-7-days prophylaxis with BAY 94-9027 in PROTECT VIII [abstract no. PB 1780]. Res Pract Thromb Haemost. 2017;1(Suppl 1):786-7.

34. Santagostino E, Kerlin BA, Negrier C, et al. Characteristics of bleed-free patients on every-5-day dosing in the PROTECT VIII (BAY 94-9027) study [abstract]. Blood. 2018;132(Suppl 1):2486.

35. Linardi C, Enriquez MM, Lalezari S. BAY 94-9027 prophylaxis improves quality of life: Haemo-QoL-A data from the PROTECT VIII study [abstract no. W-P-010 (737)]. Haemophilia. 2018;24(Suppl 5):21-2.

36. Kerlin BA, Simpson ML, Reding MT, et al. Comparison of bleeding rates before and during BAY 94-9027 prophylaxis: data from the PROTECT VIII study and extension [abstract plus poster]. In: 4th Biennial Summit of the Thrombosis and Hemostasis Societies of North America. 2018.

37. Pabinger I, Oldenburg J, Huth-Kuhne A, et al. Prophylaxis with extended dosing of BAY 94-9027 decreases overall and joint bleeding rates with consistent consumption for over 4 years of treatment [abstract no. P09-5]. In: Annual Meeting of the Society of Thrombosis and Haemostasis Research. 2019.

38. Reding MT, Ng HJ, Tseneklidou-Stoefer D, et al. Safety of longterm prophylaxis with BAY 94-9027: interim results of $>5$ years of treatment in the PROTECT VIII extension trial [abstract no. W-P-001 (404)]. Haemophilia. 2018;24(Suppl 5):17-8.

39. Hanley J, McKernan A, Creagh MD, et al. Guidelines for the management of acute joint bleeds and chronic synovitis in haemophilia: a United Kingdom Haemophilia Centre Doctors' Organisation (UKHCDO) guideline. Haemophilia. 2017. https://doi. org/10.1111/hae.13201. 
40. Schwartz CE, Powell VE, Su J, et al. The impact of extended halflife versus conventional factor product on hemophilia caregiver burden. Qual Life Res. 2018;27(5):1335-45.

41. Baxalta US Inc. ADYNOVATE ${ }^{\circledR}$ : US prescribing information. 2016. https://www.fda.gov/. Accessed 6 June 2019.

42. European Medicines Agency. Rurioctocog alfa pegol (Adynovi ${ }^{\circledR}$ ): EU summary of product characteristics. 2019. https://www.ema. europa.eu/. Accessed 6 June 2019.

43. Novo Nordisk Inc. ESPEROCT ${ }^{\circledR}$ [antihemophilic factor (recombinant), glycopegylated-exei]: US prescribing information. 2019. https://www.fda.gov/. Accessed 6 June 2019.
44. Ayyagari R, Deschaseaux C, Vashi PB, et al. Matching-adjusted indirect comparisons (MAICs) of efficacy and consumption of bay 94-9027 versus three recombinant factor VIII (rFVIII) for prophylaxis of severe hemophilia A [abstract no. PSY12]. Value Health. 2018;21(Suppl 1):S246.

45. Shah A, Solms A, Wiegmann S, et al. BAY 94-9027 and recombinant factor VIII Fc fusion protein: a head-to-head, randomized, crossover, pharmacokinetic study in patients with severe haemophilia A [abstract no. P020]. Haemophilia. 2019;25(Suppl 1):45. 